\title{
Acute isolated anterior cerebral artery infarcts: A clinical - radiological study
}

\author{
${ }^{1}$ Sanjith Aaron $M D D M,{ }^{2}$ Divyan Pancharatnam $M D,{ }^{1}$ Amal M Al Hashmi $M D$ FRCPC \\ Central Stroke Unit, Neuroscience Directorate \& ${ }^{2}$ Radiology Department, Khoula Hospital, Muscat, \\ Oman
}

\begin{abstract}
Background: The anterior cerebral artery (ACA) supplies many eloquent areas and can have anatomical variations making ACA strokes clinically and radiologically challenging. This study looks at the clinical and radiological features of isolated acute ACA strokes from a stroke centre in Oman. Methods: A retrospective study conducted over a 2 year period on ACA strokes presenting within 12 hours of symptom onset. TOAST classification was used for aetiology. National Institutes of Health Stroke Scale (NIHSS) and Modified Rankin Scale (mRS) were used to assess stroke severity. Fischer's classification was used for assessing the arterial segments with CT angiogram. Heidelberg Bleeding Classification was used for haemorrhagic conversion. Results: Isolated ACA strokes constituted 25/1180 (2.1\%) of ischemic strokes. Males 15/25 (60\%) Mean age was 68.4 years (Range $42-97$ years). Twenty eight percent of patients had earlier strokes. Hemiparesis (68\%) was the commonest clinical presentation. Twenty percent had only lower limb weakness. Hypertension 22/25 (88\%) followed by diabetes mellitus 12/25 (48\%) was the commonest risk factor. The mean NIHSS was 9 (range 3 to 13). In $36 \%$ of patients there was progression of stroke. Plain CT Picked the infarct only in 6/24 (25\%). Left side involvement in 18/25 (72\%) Artery of Hubner was involved in 6/25 (24\%); 44\% had an embolic aetiology. There was no mortality and at discharge, 11/25 (44\%) had mRS3 or less.

Conclusions: In acute ACA infarcts a CT scan can miss the diagnosis in 74\%. An embolic aetiology has to be considered in any Isolated ACA stroke and the outcome appears to be good.
\end{abstract}

Keywords: Anterior cerebral artery, acute ischemic stroke, isolated anterior cerebral artery infarct, anatomical variations, clinical presentations, imaging in ischemic strokes, radiological features

\section{INTRODUCTION}

The anterior cerebral artery (ACA) is one of the oldest arteries from the evolutionary aspect, formed as the terminal branch of the carotid artery. Phylogenetically it supplies the olfactory lobes in fish and amphibians and forebrain of reptiles and birds. When the frontal lobes evolved in mammals and humans the ACA also evolved branches and anastomosis to supply superior and medial frontal areas. ${ }^{1}$ The ACA supplies many eloquent areas and an ACA infarction can have varying clinical presentation depending on the segment involved. To make this more complicated, there is great variation in its blood supply with many anatomical variations which make ACA strokes clinically and radiologically challenging. ${ }^{2,3}$

Isolated ACA infarcts are uncommon and accounts for $1.1 \%$ to $2.3 \%$ of ischemic strokes.-6 Many registries and series have reported embolic occlusions as an important cause for ACA Strokes.,7-8 In a report from Japan, dissection was found to be an important pathogenic aetiology ${ }^{9-10}$ In this study we looked at the varying clinical presentations and radiological features and aetiology of isolated ACA strokes. Also the anatomical variations of ACA and its main branches and their possible impact on the clinical and radiological features and outcome were explored.

\section{METHODS}

This study was conducted in the in the stroke unit of one of the referral centres for stroke in the Sultanate of Oman. Patients with isolated ACA Strokes over a period of 2 years (form January 2018 to December 2019) were identified from the hospitals electronic medical records and the radiological images were reviewed from picture 
archiving and communication system. Patient demographics and clinical details were noted. National Institutes of Health Stroke Scale (NIHSS) and Modified Rankin Scale (mRS) were used to assess stroke severity at admission and discharge. ${ }^{11}$ The TOAST Criteria ${ }^{12}$ was used to classify the stroke aetiology.

All the radiological images were reviewed by radiologists. We followed the Fischer's classification $^{13}$ that divided the ACA into 5 segments: Precommunicating (A1); the vertical segment below the genu of the corpus callosum (A2); the segment curving around the genu of the corpus callosum (A3); the terminal branches (A4); and the (A5) was used to identify the arterial segment involved. Haemorrhagic conversion of the infarcted areas were classified according to the Heidelberg Bleeding Classification. ${ }^{14}$ Anatomical variants of ACA was noted as described by the CT angiogram based study by Sosa et al. ${ }^{15}$ Two-tailed Fisher's exact test was used to look for significant associations. Statistical analysis was done using a statistical package STATA (StataCorp).

\section{RESULTS}

During the 2 year period a total of 1,180 cases of ischemic strokes were treated at the centre, out of these $25(2.1 \%)$ were patients with isolated ACA strokes. All patient were seen within 12 hours of their symptom onset. Six patients arrived within the window period for thrombolysis ( $<4.5$ hours), with 5/6 received IV thrombolysis. One patient had an earlier stroke and the initial CT and CT perfusion did not show any features of acute stroke and hence not thrombolysed. None of the patients had large vessel occlusion. Therefore none had thrombectomy. Males constituted 15/25 (60\%). The mean age was 68.4 years (Range 42 - 97 years). Hypertension was the commonest risk factor seen in $22 / 25(88 \%)$ followed by diabetes mellitus $12 / 25(48 \%)$ and dyslipidemia 6/25 (24\%); 4/25 $(16 \%)$ were current smokers and another $4 / 5$ (16\%) were patient with known atrial fibrillation. Seven $(7 / 25,28 \%)$ patients had earlier strokes and $5 / 25(20 \%)$ were on treatment for ischemic heart disease. Hemiparesis and hemiplegia were the commonest clinical presentation in $17 / 25$ $(68 \%)$. In $6 / 25(24 \%)$ the hemiparesis was associated with confusion and in another 5/25 (20\%) there was associated dysarthria. In 5/25 (20\%) there was only lower limb weakness 3 (12\%) patients presented only with confused behaviour. Associated urinary incontinence was noted in one patient with hemiparesis.
The mean NIHSS was 9 (Range 3 -13); 6 patients had residual deficits form earlier strokes and 3 patients were bed bound due to dementing illness. In 9/25 (36\%), the patients had progression of symptoms and recruitment of new deficits after admission.

In 24/25 (96\%) patients the first investigation was plain CT (with CT Perfusion in 7/24). The plain CT picked up the ACA infarct only in 6/24 (25\%) (One patient had MRI in the acute phase). (Figure 1)

The CT perfusion picked up the infarct in $4 / 7(57 \%)$. In the remaining a repeat CT done between 24 To 72 hours (7 patients) and MRI DWI sequence (7 patients) was needed to show the infarct. (Figure 2)

The infarct was on the left side in 18/25 (72\%) and on the right in 5. Two patients had bilateral involvement.

Vascular imaging was done in 17/25 (68\%). The variants in the anterior communicating artery (A-Com) were as noted in Figure 3: $-\mathrm{X}$ shaped A-Com in $2(12 \%)$, absence of A-Com and a Y shaped A-Com in one each (6\%). In the remaining $13(76 \%)$, the A-Com had a typical configuration. Hypoplasia of the A1 segment was seen in 4 (24\%) and a total absence and fenestrated A1 was seen in one each (6\%). As for ACA A2 segment, hypoplasia was seen in 2 patients and one each had total absence and a triple A2 segment. Artery of Hubner was involved in 6/25 (24\%) patients, and in these the A2 segment was involved in 4 and $\mathrm{A} 1$ in one.

Eight (8/25) patients had carotid atherosclerotic disease; 3 ipsilateral to the ACA infarct. However none had a stenosis of $>70 \%$. Hemorrhagic conversion of the infarcts was seen in 4 patient. Based on the Heidelberg bleeding classification, 2 patients had $\mathrm{H} 1$, one each $\mathrm{H} 2$ and $\mathrm{PH} 2$. There was history of earlier strokes in 7/25 (28\%). These earlier strokes were in the ipsilateral middle cerebral artery (MCA) territory in 4, contralateral MCA in 2 and in the posterior circulating in 1. 6/25 (24\%) had silent infarcts (ipsilateral MCA 2 contralateral MCA 2 and posterior cerebral artery 2). Five patients (20\%) were treated by IV thrombolysis. Based on the TOAST Criteria (Table 1), the majority of the patients $11 / 25(44 \%)$ had an embolic stroke. Of the 7 patients who had atrial fibrillation, 4 were already known cases and of these 2 had their INR in sub-therapeutic range and one patient had missed medications for 3 days at the time of stroke. Three patients were found to have atrial fibrillation after their stroke. 


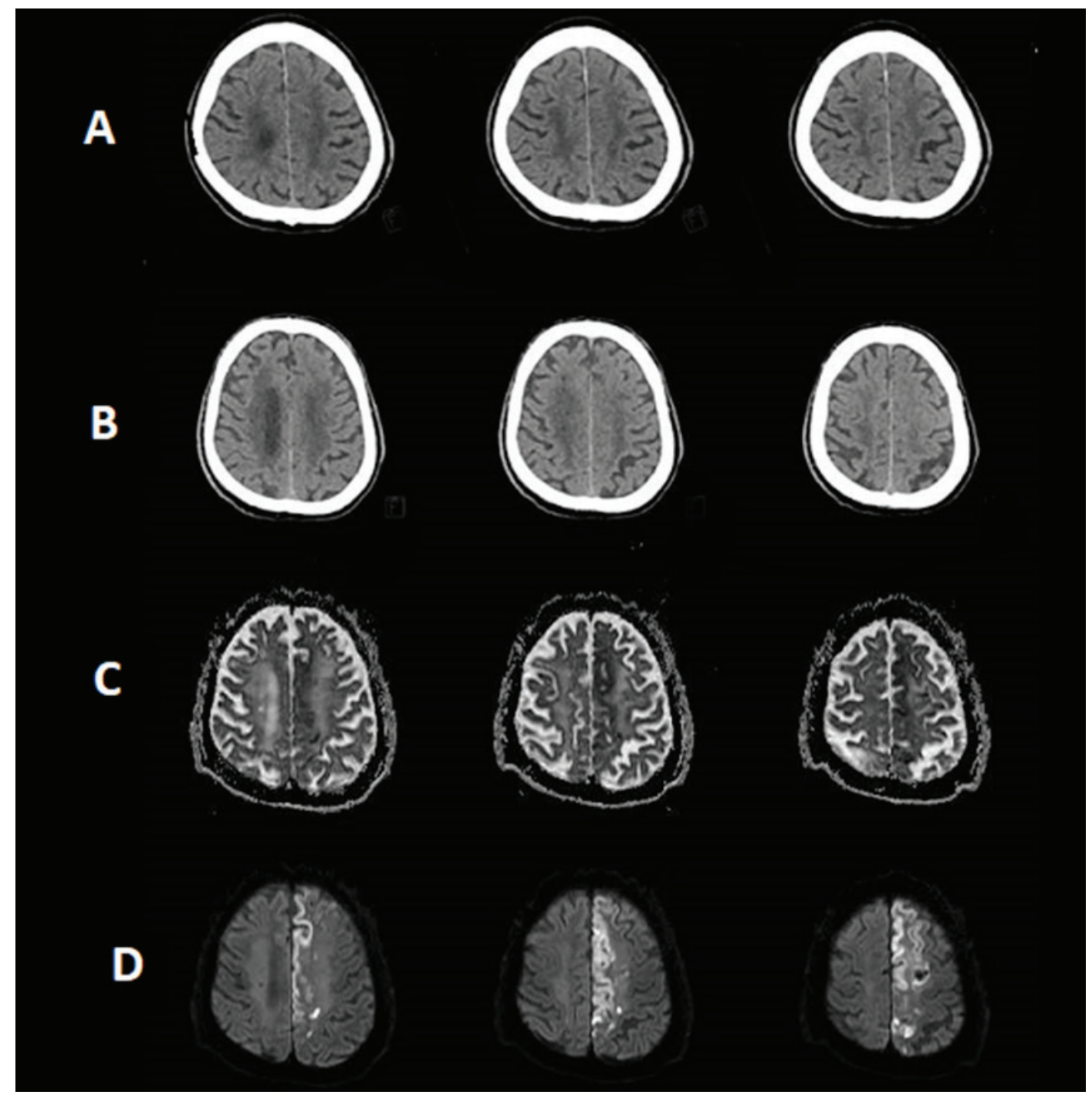

Figure 1. Plain CT scan Images (A) Day 1 and (B) Day 2 not showing any infarcts and MRI (C) ADC and (D) DWI Sequences done on day 4 showing infarction in the left ACA territory

As for complications, 6/25 had severe dysphagia requiring RT Feeds. Complications in the acute phase were aspiration pneumonia $3 / 25$. Early post stroke seizures $3 / 25$, Urinary tract infection. On patient developed congestive cardiac failure needing ventilator support. At the time of discharge $11 / 25$ patient had mRS of 3 or less. In the remaining, 14/25 who had a mRS of 4 and 5, 7 patients had earlier strokes and in 4 of them base line MRIS was already 3 .

At 3 month follow up, 2 patients died, one due to severe CCF and another due to severe sepsis and shock. The rest had either full recovery or mild residual disability.

\section{DISCUSSION}

In our series the incidence of isolated ACA stroke was $2.1 \%$ of the ischemic strokes, which was similar to that described in the literature. ${ }^{4-6}$ All the patients had presented within 12 hours of their symptoms, however a plain CT scan which is usually the first investigation for suspected acute strokes could detect the ACS infarct in only $25 \%$. (Figure 1,2 ) This is significant since in $20 \%$ the presentation was only with isolated lower limb weakness and a normal CT may lead to the erroneous localization to a pathology outside the CNS and in another $12 \%$ the presenting complaint was behavioral changes which again with a normal scan could make the clinician think of an alternate diagnosis. 


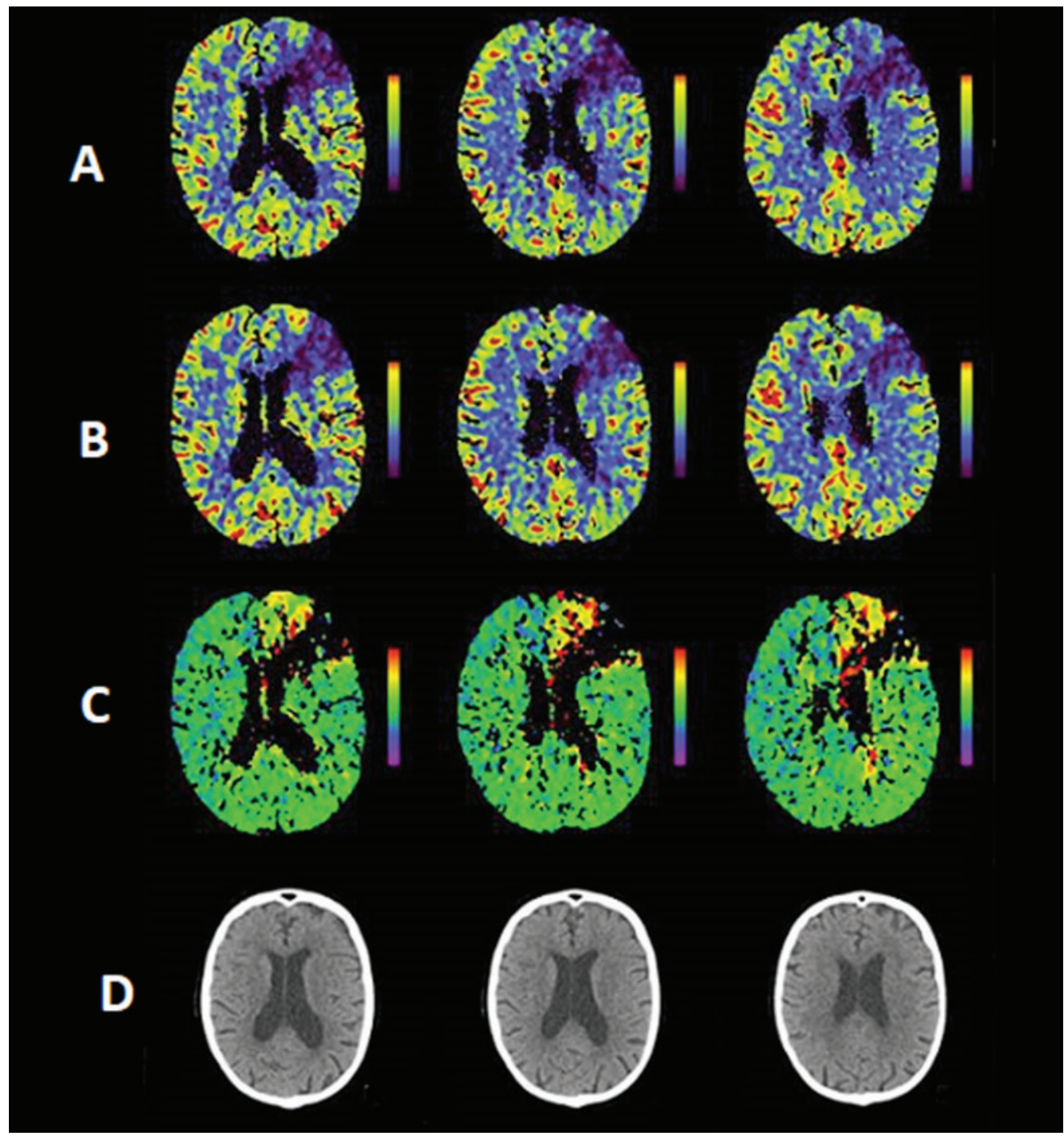

Figure 2. CT perfusion images (A) Cerebral blood flow; (B) Cerebral blood volume; and (C) Time to peak showing matched deficit in the left ACA territory. The CT axial sections (D) done concurrently showing no features to suggest any infarcts.

The mean NIHSS was 9 with a range varying from 3 to 13 . However all the patient who had NIHSS of more than 10 had either an earlier stroke or dementia with residual deficits which had contributed to the high NIHSS. If we exclude these patient, the mean NIHSS was only 5. Even though the ACA supply the leg areas, hemiparesis and hemiplegia were the commonest clinical presentation in $68 \%$. However the lower limb weakness was more pronounced compared with the upper limbs.

The artery of Hubner was involved in $24 \%$ of the patients. All except one had significant dysarthria as their initial presenting symptom apart for the hemiparesis, and one patient had choreiform movements involving the upper limb.
It is well documented in the literature that embolism is an important etiology in ACA strokes. ${ }^{16-17}$ A cardiac embolic source was noted in $44 \%$ of our cases. (Table 1) In addition; 3 patients had carotid atherosclerotic disease ( $<70 \%$ stenosis) ipsilateral to the infarct raising the possibility of artery of artery thromboembolism. However some regional variation in the etiology has been reported. Kazui et al.$^{18}$ reported that in the Japanese population, the etiology of isolated ACA infarcts are mainly atherosclerotic rather than embolic. This may be a reflection of the overall higher prevalence of intracranial atherosclerotic disease in the East as compared to the West. It is also interesting to note that the left ACA 


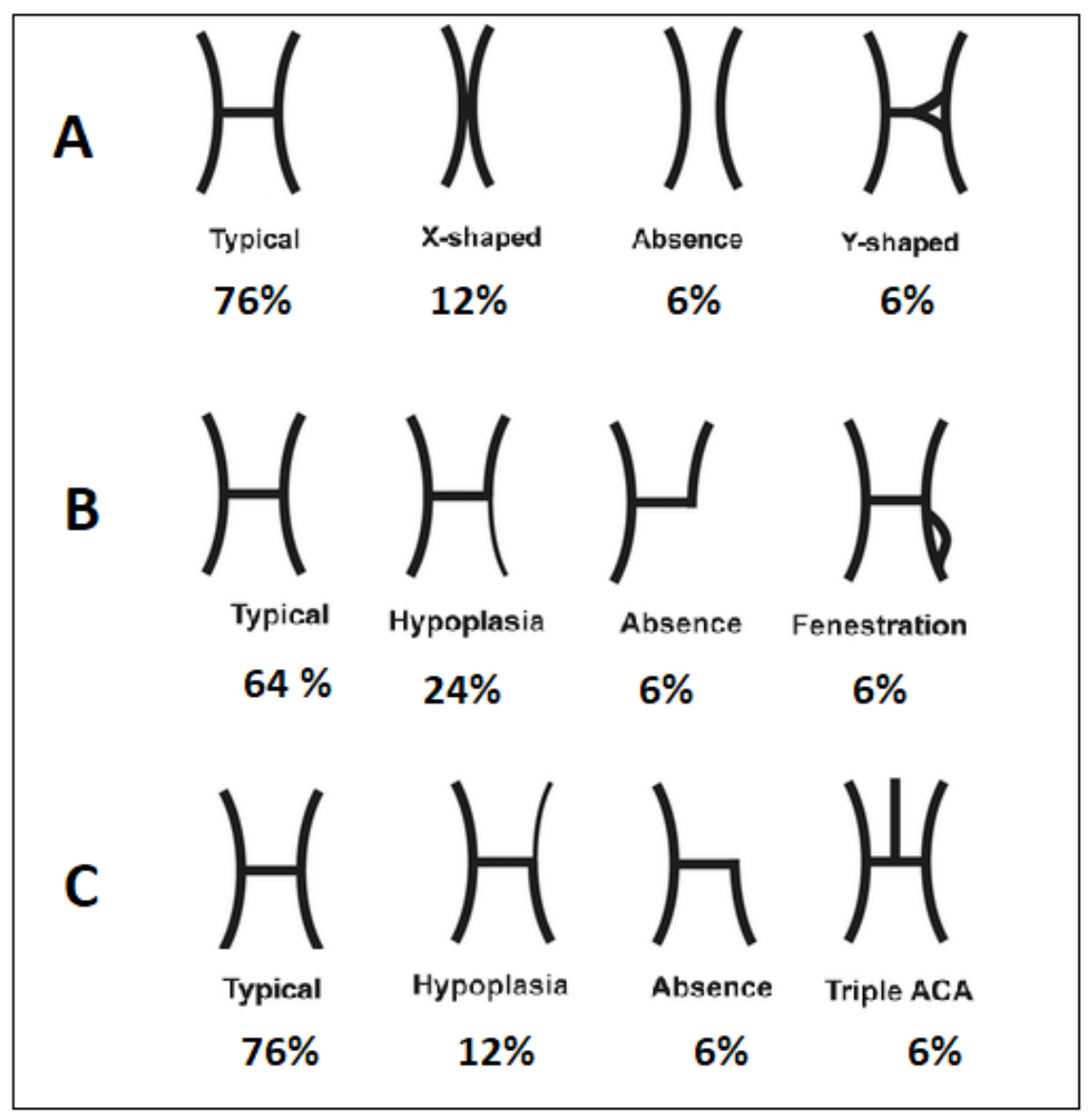

Figure 3. Variants seen in the (A) A-Com, (B) A1 segment, and (C) A2 segment of ACA.

was involved in $72 \%$ of our cases. It has been documented in the literature that cardiac emboli tends to have a predilection for the left internal carotid artery and its branches especially in the setting of atrial fibrillation. ${ }^{16,17}$ Embolic stroke due to unstable aortic arch atheroma also tend to cause more left hemispheric strokes. ${ }^{19}$ However there was no evidence of any significant aortic arch atheroma in any of our patients.

Also the findings of earlier stroke in $28 \%$ and silent infarcts in $24 \%$ emphasizes the importance to actively investigate for an embolic source in

Table 1: Aetiology according to the TOAST criteria

\begin{tabular}{lc}
\hline \multicolumn{2}{c}{ Etiology According to the TOAST Criteria } \\
\hline Large artery atherosclerosis & 9 \\
\hline Intracranial atherosclerosis & 9 \\
\hline Cardio embolic & 11 \\
\hline Atrial fibrillation & 7 (1 With LA Thrombus) \\
\hline Dilated ischemic cardiomyopathy & 4 \\
\hline Other determined etiology & 2 \\
\hline Dissection & 1 \\
\hline Vasculitis & 3 \\
\hline Undetermined etiology & 3 \\
\hline Evaluation incomplete &
\end{tabular}


any patient with an ACA stroke. Two patient had bilateral ACA infarcts and their vascular imaging showed hypoplasia of A1 segment one side. Studies have shown that hypoplasia or absence of one A1 segment can be seen in up to $11 \%$ and bilateral ACA territories can be involved either with embolic or atherosclerotic occlusion of the dominant A1 Segment in these variants. ${ }^{15}$

Fused or X shaped A-Com was the commonest variation in the A-Com seen. Complete absence of A-Com in 6\% and hypoplasia of A1 and A 2 segments were similar to seen in the literature. ${ }^{20-22}$ Isolated ACA infarcts are known to have a good short term outcome. ${ }^{23}$ This is reflected in our series where there was no in-hospital mortality, however in the follow up period 2 patients died as a consequence of non-neurological causes.

In conclusion, this study highlights firstly, in the acute setting ACA strokes can be missed on plain CT. Secondly, in a patient presenting with ACA stroke, one need to consider an embolic etiology and to extensively investigate for the same for proper preventive treatment.

\section{REFERENCES}

1. Lasjaunias P, Berenstein A, Ter Brugge K. Clinical vascular anatomy and variations. In: Lasjaunias $\mathrm{P}$, Berenstein A, Ter Brugge K, eds: Surgical neuroangiography: 2nd ed. Vol 1. Berlin: SpringerVerlag Berlin and Heidelberg GmbH \& Co.K. 2001.

2. van der Zwan A, Hillen B, Tulleken CA, Dujovny M, Dragovicet L. Variability of the territories of the major cerebral arteries. J Neurosurg 1992;77(6):92740.

3. Lemay M, Gooding CA. The clinical significance of the azygos anterior cerebral artery (ACA). Am J Roentgenol 1966;98(3):602-10.

4. Vemmos KN, Takis CE, Georgilis K, et al. The Athens stroke registry: results of a five-year hospital-based study. Cerebrovasc Dis 2000; 10:133-41.

5. Ghandehari K, Izadi Z. The Khorasan Stroke Registry: results of five-year hospital-based study. Cerebrovasc Dis 2007; 23: 132-9.

6. Kumral E, Bayulkem G, Evyapan D, Yunten N. Spectrum of anterior cerebral artery territory infarction: clinical and MRI findings. Eur J Neurol 2002; 9:615-24.

7. Arboix A, García-Eroles L, Sellarés N, Raga A, Oliveres M, Massons J. Infarction in the territory of the anterior cerebral artery: clinical study of 51 patients. BMC Neurol 2009;9:30.

8. Moulin T, Tatu L, Vuillier F, Berger E, Chavot D, Rumbach L. Role of a stroke data bank in evaluating cerebral infarction subtypes: patterns and outcome of 1,1776 consecutive patients from the Besançon Stroke Registry. Cerebrovasc Dis 2000; 10:261-71.

9. Toyoda K. Anterior cerebral artery and Heubner's artery territory infarction. Front Neurol Neurosci 2012;30:120-2.
10. Hensler J, Jensen-Kondering U, Ulmer S, Jansen O. Spontaneous dissections of the anterior cerebral artery: a meta-analysis of the literature and three recent cases. Neuroradiology 2016; 58(10):997-1004.

11. van Swieten J C, Koudstaal PJ, Visser M C, et al. Interobserver agreement for the assessment of handicap in stroke patients. Stroke 1988; 19(5):604-7.

12. Goldstein LB, Jones MR, Matchar DB, et al. Improving the reliability of stroke subgroup classification using the Trial of ORG 10172 in Acute Stroke Treatment (TOAST) criteria. Stroke 2001; 32(5):1091-8.

13. Lasjaunias P, Brugge KGT, Berenstein A. Intracranial aneurysm in children. In: Surgical neuroangiography. Vol 3. Clinical and interventional aspect in children. Springer Berlin: 2006.

14. von Kummer R, Broderick JP, Campbell BC, et al. The Heidelberg Bleeding Classification: classification of bleeding events after ischemic stroke and reperfusion therapy. Stroke 2015; 46:2981-6.

15. Jiménez-Sosa MS, Cantu-Gonzalez JR, MoralesAvalos R, et al. Anatomical variants of anterior cerebral arterial circle: A study by multidetector computerized 3D tomographic angiography. Int $J$ Morphol 2017; 35(3):1121-8.

16. Moulin T, Tatu L, Vuillier F, Berger E, Chavot D, Rumbach L. Role of a stroke data bank in evaluating cerebral infarction subtypes: patterns and outcome of 1,1776 consecutive patients from the Besançon Stroke Registry. Cerebrovasc Dis 2000; 10:261-71.

17. Arboix A, García-Eroles L, Sellarés N, Raga A, Oliveres M, Massons J. Infarction in the territory of the anterior cerebral artery: clinical study of 51 patients. BMC Neurol 2009;9:30.

18. Kazui S, Sawada T, Naritomi H, Kuriyama Y, Yamaguchi T. Angiographic evaluation of brain infarction limited to the anterior cerebral artery territory. Stroke 1993;24:549-53.

19. Elsaid N, Bigliardi G, Dell'Acqua ML, et al. The relation between aortic arch branching types and the laterality of cardio-embolic stroke. J Stroke Cerebrov Dis 2020; 29(7); 104917.

20. Rhoton AL Jr, Saeki N, Perlmutter D, Zeal A. Microsurgical anatomy of common aneurysm sites. Clin Neurosurg 1979; 26: 248-306.

21. Kardile PB, Ughade JM, Pandit SV, Ughade MN. Anatomical variations of anterior communicating artery. J Clin Diagn Res 2013; 7(12):2661-4.

22. Kovač JD, Stanković A, Stanković D, Kovač B, Šaranović D. Intracranial arterial variations: a comprehensive evaluation using CT angiography. Med Sci Monit 2014;20:420-7.

23. Kumral E, Bayulkem G, Evyapan D, Yunten N. Spectrum of anterior cerebral artery territory infarction: clinical and MRI findings. Eur J Neurol 2002; 9:615-24. 